\title{
Quasi-Compactness in Quasi-Banach Spaces $\ell_{p}$, for $0<p<1$ \\ Raheam A. Mansor Al-Saphory ${ }^{1}$ Mahmood K Jasim² \\ ${ }^{1}$ Department of Mathematics \\ College of Education, Tikrit University, Iraq. \\ Saphory@hotmail.com \\ ${ }^{2}$ Department of Mathematics and Physical Sciences \\ College of Arts \& Sciences, University of Nizwa, Oman. \\ mahmoodkhalid@unizwa.edu.om
}

\section{ABSTRACT}

Quasi-compactness in a quasi-Banach space for the sequence space $\ell_{p}, 0<p<1$ has been introduced based on the important extension of Milman's reverse Brunn-Minkowiski inequality by Bastero et al. in 1995. Moreover, Many interesting results connected with quasi-compactness and quasi-completeness in a quasi-normed space $\ell_{p}$, for $0<p<1$ have been explored. Furthermore, we have shown that, the quasi-normed space under which condition is a quasi Banach space. Also, we have shown that the space if it is quasi-compact in quasi normed space then it is a quasiBanach space and the converse is not true. Finally, a sufficient condition of the existence for a quasi-compact operator from $\ell_{p} \rightarrow \ell_{p}$ has been presented and analyzed.

Keywords: Sequence space $\ell_{p}, \quad 0<p<1 ; q$-normed space; $q$-Banach space; $q$-compact space.

\section{Council for Innovative Research}

Peer Review Research Publishing System

Journal: Journal of Advances in Mathematics

\author{
Vol 4, No 1 \\ editor@cirworld.com \\ www.cirworld.com, member.cirworld.com
}




\section{INTRODUCTION}

Functional analysis is a scientific discipline of fairly recent origin. It provides a power full tool to discover solution to problems occurring in pure, applied social sciences, for instance physics, engineering, medicine, agro-industries, ecology, economics and bio-mathematics [5, 16].

One of the important notions in functional analysis is the concept of Banach space. This concept was introduced by Polish mathematician Stefan Banach in 1922 and has received much attention in the literatures ([7, 15] and references therein).

Recently, Bastero et al. extended the reverse Brunn-Minkowiski inequality which was discovered by Milman in [12] to the case of $P$-convex balls as in [10].

An extension of the Banach space concept and that is very important in practical application is that of an infinite Leslie matrix [3, 4, 13]. This problem has been introduced and developed by Al-Saphory et al. as in [3].

The purpose of this chapter is to study the notion of compactness for a quasi-Banach space for sequence space $\ell_{p}$ where $0<p<1$ and to prove some results which are relevant to this work. The main reason for introducing the notion of quasi-compactness is the possibility to construct a quasi-compact operator from $\ell_{p}$ to $\ell_{p}$, where $0<p<1$ in order to determine the growth of population [4]. This paper is organized as following:

Section 2 devotes an introduction of the sequence space $\ell_{p}, 0<p<1$, quasi- normed space and quasi-Banach space. We give the definition of sequence space $\ell_{p}$, where $0<p<1$, and we show that is not normed space, but may be a quasi-normed space. We give some interesting results concerning this notion in order to show that the space $\ell_{p}, 0<p<1$ is a quasi-Banach space. Section 3 related to explore the concept of quasi-compact sets in a quasinormed space, It also contains some examples, remarks and results which explain the relation between these concepts. In section 4, we propose the concept of an operator which is of many types such as a quasi-compact operator. Also, we present some results concerning to this concept.

\section{Quasi-Banach Space}

This section show that the space $\ell_{p}, 0<p<1$ is quasi-Banach space [3], that means, we mention the definition of sequence space $\ell_{p}, 0<p<1$, as well as, some concepts and results related to this space [2-4].

Definition 2.1 [1]: The sequence space $\ell_{p}, 0<p<1$, is the space of all sequences $x=\left\{x_{i}\right\}$ in $R$ or $C$ such that

$$
\sum_{i=1}^{\infty}\left|x_{i}\right|^{p}<\infty
$$

Remark 2.2: The sequence space $\ell_{p}, 0<p<1$, with the function

$$
\|x\|_{p}=\left(\sum_{i=1}^{\infty}\left|x_{i}\right|^{p}\right)^{1 / p}
$$

is not a normed space, because the condition (3) of the norm definition [8] is not satisfied. To explain this remark, we consider the following example:

Example 2.3: Let $\mathrm{p}=1 / 2$, and suppose $x$ and $y$ are two sequences, where

$$
x=\left\{x_{i}\right\}=\{0.1,0,0,0, \ldots\} \text { and } y=\left\{y_{i}\right\}=\{0,0.2,0,0, \ldots\} .
$$

Then we have:

$$
\|x+y\|_{1 / 2}=\left(\sum_{i=1}^{\infty}\left|x_{i}+y_{i}\right|^{1 / 2}\right)^{2}=0.5828424
$$

and

$$
\|x\|_{1 / 2}+\|y\|_{1 / 2}=\left(\sum_{i=1}^{\infty}\left|x_{i}\right|^{1 / 2}\right)^{2}+\left(\sum_{i=1}^{\infty}\left|y_{i}\right|^{1 / 2}\right)^{2}=0.3
$$


It is clear that:

$$
\|x+y\|_{1 / 2}>\|x\|_{1 / 2}+\|y\|_{1 / 2}
$$

Thus, the space $\ell_{p}, 0<\mathrm{p}<1$, is not a normed space.

Lemma 2.4 [8]: Let $\left\{x_{i}\right\}$ be any (real or complex) sequence and $0<p \leq 1$. Then,

$$
\left|\sum_{i=1}^{\infty} x_{i}\right|^{p} \leq \sum_{i=1}^{\infty}\left|x_{i}\right|^{p}
$$

Here, we present the concept of a quasi-normed space for $\ell_{p}, 0<p<1$, this notion has been introduced recently in [3]. In this paper, we usually consider the space $X=\ell_{p}, 0<p<1$.

Definition 2.5: Let $X=\ell_{p}, 0<p<1$ is a vector space over a field $F$. A quasi-norm on $X$ is a function ${ }_{q}\|\cdot\|: X \longrightarrow R_{+0}$ such that:

(1) ${ }_{q}\|x\| \geq 0, \forall x \in X,{ }_{q}\|x\|=0$ if and only if $x=0$,

(2) ${ }_{q}\|\lambda x\|=|\lambda|_{q}\|x\|, \quad \forall x \in X, \forall \lambda \in F$,

(3) There exists a constant $\gamma \geq 1$ such that,

$$
{ }_{q}\|x+y\| \leq \gamma\left({ }_{q}\|x\|+{ }_{q}\|y\|\right), \quad \forall x, y \in X
$$

The pair $\left(X,{ }_{q}\|\|.\right)$ is called a quasi-normed space. For simplifying, we denote the space ${ }_{q} X=\ell_{p}, 0<p<1$, as a $q$-normed space. For more details of concept $q$-normed space see references $[9,18]$

Proposition 2.6[3]: The sequence space $\ell_{p}, 0<p<1$, with

$$
{ }_{q}\|x\|_{p}=\left(\sum_{i=1}^{\infty}\left|x_{i}\right|^{p}\right)^{1 / p}
$$

is a $q$-normed space.

Remark 2.7: From the proposition 2.6, we note that a constant $\gamma$ in the definition 2.5 with $\ell_{p}$, where $0<p<1$, can be taken to be $2^{1 / p}$ (see ref. [3] ).

Remark 2.8: According to the definition 2.1 and the definition of the norm as in [7], a normed space $\ell_{p}, 1 \leq p<\infty$, with the norm

$$
\|x\|_{p}=\left(\sum_{i=1}^{\infty}\left|x_{i}\right|^{p}\right)^{1 / p}
$$

is a $q$-normed space. Conversely, in general, is not true and is true only if $\gamma=1$, as it is shown in the following example:

Example 2.9: Consider the sequence space $\ell_{p}, 0<p<1$, then we can deduce that, from the remark 2.2 and the proposition 2.6, this space is not normed space, but it may be a $q$-normed space.

Now, we recall the notion of a $q$-Banach space for the space $\ell_{p}, 0<p<1$ [3]. Then, we study the convergent and completeness sequence in the $q$-normed space.

Definition 2.10: Let ${ }_{q} X=\ell_{p}, 0<p<1$ be a $q$-normed space

(1) A sequence $\left\{x_{n}\right\}$ in ${ }_{q} X$ is called a $q$-convergent to a point $x \in{ }_{q} X$ if and only if 


$$
{ }_{q}\left\|x_{n}-x\right\| \rightarrow 0 \text { as } n \rightarrow \infty \text {. }
$$

(2) A sequence $\left\{x_{n}\right\}$ in ${ }_{q} X$ is a $q$-Cauchy sequence if and only

$$
{ }_{q}\left\|x_{n}-x_{m}\right\| \rightarrow 0 \text { as } n, m \rightarrow \infty \text {. }
$$

Lemma 2.11: If ${ }_{q} X$ be a $q$-normed space, then every a $q$-convergent sequence is a $q$-Cauchy sequence, but not conversely in general. Hence, $\left\{x_{n}\right\}$ is a $q$-Cauchy sequence. For the converse, we take the following example:

Example 2.12: Let ${ }_{q} X=C[-1,1]$ which is a vector space of all continuous functions defined on $[-1,1]$ with the following a quasi-norm:

$$
{ }_{q}\|f\|=\left(\int_{-1}^{1}|f(x)|^{2} d x\right)^{1 / 2} \quad,(f \in C[-1,1])
$$

Consider the sequence $\left\{f_{n}\right\}$ in $C[-1,1]$, defined as follows:

$$
f_{n}(x)= \begin{cases}0 & ,-1 \leq x \leq 0 \\ n x & , 0<x \leq 1 / n \\ 1 & , 1 / n<x \leq 1\end{cases}
$$

Then $\left\{f_{n}(x)\right\}$ is a $q$-Cauchy sequence in space $C[-1,1]$, but it is not a $q$-convergent to an element of this space [14].

Definition 2.13: A sequence $\left\{x_{n}\right\}$ in a $q$-normed space ${ }_{q} X$ is called a $q$-bounded sequence if and only if there exists a positive real number $M$ such that ${ }_{q}\left\|x_{n}\right\| \leq M$ for all $n \in N$.

Theorem 2.14 [3]: Let ${ }_{q} X$ be a $q$-normed space, then

(1) Every a $q$-Cauchy sequence is a $q$-bounded.

(2) A $q$-convergent sequence has a unique limit.

(3) A $q$-Cauchy sequence is a $q$-convergent if and only if it has a $q$-convergent sub-sequence.

Definition 2.15: Let $\left\{x_{n}\right\}$ be a sequence in a $q$-normed space ${ }_{q} X$

(1) A series $\sum_{n=1}^{\infty} x_{n}$ is called a $q$-convergent to $s \in{ }_{q} X$ if and only if

$$
{ }_{q}\left\|S_{n}-s\right\| \rightarrow 0, \text { as } n \rightarrow \infty,
$$

where $S$ is the limit of $\left\{S_{n}\right\}$, otherwise, it is a $q$-divergent.

(2) A series $\sum_{n=1}^{\infty} x_{n}$ is called absolutely $q$-convergent if and only if

$$
\sum_{n=1}^{\infty}{ }_{q}\left\|x_{n}\right\|<\infty
$$

Thus, we study the relation between $q$-Cauchy sequence and $q$-convergent sequence in $q$-Banach space.

Definition 2.16: A $q$-normed space, in which every $q$-Cauchy sequence is $q$-convergent, is called $q$-Banach space.

Remark 2.17: Now we can deduce that, the Banach space $\ell_{p}, 1 \leq p \leq \infty$ [13], is $q$-Banach space. But the converse may not be true, as it is shown in the following example: 
Example 2.18: The sequence space $\ell_{p}, 0<p<1$ is $q$-Banach space, which will be proved in the theorem 2.20. But, it is not Banach space by remark 2.2.

Theorem 2.19 [3]: A q-normed space ${ }_{q} X$ is a complete if and only if every absolutely a $q$-convergent series in ${ }_{q} X$ is also a $q$-convergent in ${ }_{q} X$.

Theorem 2.20 [3]: The sequence space $\ell_{p}, 0<p<1$, is a $q$-Banach space.

Remark 2.21: We can deduce that any Banach space is a $q$-Banach space.

Lemma 2.22 [11]:Let $x$ be a non-negative real number. If $0 \leq x<1$, then

$$
\sum_{n=0}^{\infty} x^{n}=\frac{1}{1-x}
$$

and if $x \geq 1$, the series diverges.

Definition 2.23: Let $\left\{x_{n}\right\}$ be a sequence in a $q$-normed space ${ }_{q} X$.

(a) A series $\sum_{n=1}^{\infty} x_{n}$ is called $q$-convergent to $s \in X$ if and only if ${ }_{q}\left\|S_{n}-s\right\| \rightarrow 0$, as $n \rightarrow \infty$, where $s$ is the limit of $\left\{S_{n}\right\}$. Otherwise, it is $q$-divergent.

(b) A series $\sum_{n=1}^{\infty} x_{n}$ is called absolutely $q$-convergent if and only if

$$
\sum_{n=1}^{\infty}\left\|x_{n}\right\|<\infty
$$

\section{Quasi-Compact sets in the Space $\ell_{p}$, for $0<p<1$}

In this section, we introduce the concept of quasi compact sets in a $q$-normed space with some definitions and results which are relevant to this work.

Definition 3.1: Let ${ }_{q} X$ be a $q$-normed space. All points and sets mentioned below are understood to be elements and subsets of ${ }_{q} X$.

(a) A q-open ball of a point $x$ is a set ${ }_{q} B_{r}(x)$ consisting of all points $y$ such that ${ }_{q}\|x-y\|<r$. The number $r$ is called the radius of ${ }_{q} B_{r}(x)$ where $r>0$.

(b) A point $x$ is an interior point of the set $E$ if there is a $q$-open ball ${ }_{q} B_{r}(x)$ such that ${ }_{q} B_{r}(x) \subset E$.

(c) $\quad E$ is a $q$-open set if every point of $E$ is an interior point of $E$.

(d) A point $x$ is a limit point of $E$ if every is a $q$-open ball of $x$ contains a point $y \neq x$ such that $y \in E$. The set of all limit points of $E$ is called the $q$-derived set of $E$ and denoted by ${ }_{q} E^{\prime}$

(e) $\quad E$ is a $q$-closed set if every limit point of $E$ is a point of $E$ in the sense of definition 3.1 (d).

(f) The $q$-closure of $E$ which is denoted by ${ }_{q} \bar{E}$, is a $q$-closed set containing $E$ which is formed by adding ${ }_{q} E^{\prime}$.

(g) $E$ is a $q$-bounded set if there is a positive real number $M$ such that ${ }_{q}\|x\| \leq M$ for all $x \in E$.

(h) The complement of $E$ (denoted by $E^{c}$ ) is the set of all points $x \in X$ such that $x \notin E$.

Lemma 3.2: If ${ }_{q} X$ is a $q$-normed space and $E$ is a subset of ${ }_{q} X$, then,

(a) every $q$-open ball is an $q$-open set. 
(b) $E={ }_{q} \bar{E}$ if and only if $E$ is a $q$-closed set.

Proof:

(a) Suppose ${ }_{q} B_{r}(x)$ is a $q$-open ball of a point $x$, where $x \in{ }_{q} X$ and let $y$ be any point of ${ }_{q} B_{r}(x)$. Then by choosing a positive real number $r^{\prime}$ such that:

$$
r^{\prime}=\frac{r-c_{q}\|x-y\|}{c}, \quad \text { where } c \geq 1
$$

For all points $z$ such that ${ }_{q}\|y-z\|<r^{\prime}$, we have then

$$
\begin{gathered}
{ }_{q}\|x-z\| \leq c\left({ }_{q}\|x-y\|+{ }_{q}\|y-z\|\right) \\
<c\left({ }_{q}\|x-y\|+r^{\prime}\right)=r
\end{gathered}
$$

so that $z \in{ }_{q} B_{r}(x)$. Thus, $y$ is an interior point of ${ }_{q} B_{r}(x)$. Hence, ${ }_{q} B_{r}(x)$ is a $q$-open set.

(b) Suppose $={ }_{q} \bar{E}$. Since ${ }_{q} \bar{E}$ is a $q$-closed set by definition 3.1 (f), then, $E$ is a $q$-closed set. Conversely, if $E$ is a $q$-closed set then all limit points of $E$ belong to $E$ by definition 3.1.(e). Hence by definition 3.1(f), we have ${ }_{q} \bar{E}=E$.

Lemma 3.3 [14]: A set $E$ is $q$-closed if and only if its complement is $q$-open.

Theorem 3.4: Let $E$ be a non-empty subset of a $q$-normed space ${ }_{q} X$ and ${ }_{q} \bar{E}$ its $q$-closure of $E$, then,

(a) $x \in{ }_{q} \bar{E}$ if and only if there is a sequence $\left\{x_{n}\right\}$ in $E$ such that ${ }_{q}\left\|x_{n}-x\right\| \rightarrow 0$ as $n \rightarrow \infty$

(b) $\quad E$ is a $q$-closed if and only if there is a sequence $\left\{x_{n}\right\}$ in $E$ such that ${ }_{q}\left\|x_{n}-x\right\| \rightarrow 0$ as $n \rightarrow \infty$, implies that $x \in E$.

Proof:

(a) Let $\in{ }_{q} \bar{E}$. If $x \in E$ then a sequence of that type is $(x, x, \ldots)$. If $x \notin E$ then it is a limit point of $E$. Hence for each $n=1,2, \ldots$ the $q$-open ball ${ }_{q} B_{1 / n}(x)$ contains an $x_{n} \in E$ and $\left\|x_{n}-x\right\| \rightarrow 0$, because $1 / n \rightarrow 0$ as $n \rightarrow \infty$. Conversely, if $\left\{x_{n}\right\}$ is in $E$ and ${ }_{q}\left\|x_{n}-x\right\| \rightarrow 0$ then $x \in E$ or every $q$-open ball contains points $x_{n} \neq x$, so that $x$ is a limit point of E. Hence by definition 3.1.(f), $x \in{ }_{q} \bar{E}$.

(b) By lemma. 3.2 (b), $E$ is $q$-closed if and only if $E={ }_{q} \bar{E}$, so that (b) follows from (a).

Theorem 3.5: A subspace $E$ of a complete $q$-normed space ${ }_{q} X$ is itself complete $q$-normed space if and only if the set $E$ is $q$-closed in ${ }_{q} X$.

Proof: Let $E$ be complete. By theorem 3.4 (a), for every $x \in{ }_{q} \bar{E}$ there is a sequence $\left\{x_{n}\right\}$ in $E$ which converges to $x$. Since $\left\{x_{n}\right\}$ is a $q$-Cauchy sequence by lemma 2.11 and $E$ is complete, $\left\{x_{n}\right\} q$-converges in $E$ and the limit being unique by lemma 2.15 (b). Hence, $x \in E$. this proves that $E$ is $q$-closed because $x \in{ }_{q} \bar{E}$ was arbitrary.

Conversely, let $E$ be closed and $x_{n}$ is a $q$-Cauchy sequence in $E$. Then

$$
{ }_{q}\left\|x_{n}-x\right\| \rightarrow 0 \text { and } x \in X
$$

which implies $x \in{ }_{q} \bar{E}$ by theorem 3.4 (a) and $x \in E$, since $E={ }_{q} \bar{E}$ by assumption. Hence the arbitrary $q$-Cauchy sequence $x_{n}$, which $q$-converges in $E$, which proves completeness of $E$.

Remark 3.6: According to the theorems 2.20 and 3.5, a $q$-Banach space 


$$
\ell_{p}, 0<p<1 \text { is } q \text {-closed. }
$$

Definition 3.7: Let $E$ be a subset of a $q$-normed space ${ }_{q} X$. The $q$-diameter of $E$ which is denoted by $d(E)$ is defined as:

$$
{ }_{q} d(E)=\sup \left\{{ }_{q}\|x-y\| \mid x, y \in E\right\}
$$

Definition 3.8: Let ${ }_{q} X$ be a $q$-normed space and $E_{n}, n \in N$ is a non-empty $q$-closed subset of $X$. A $q$-nest of $q$ closed sets is a sequence $\left\{E_{n}\right\}$ of $q$-closed sets $E_{n}$ such that $E_{n+1} \subset E_{n}$ for each $n$ and $\lim _{n \rightarrow \infty} q\left(E_{n}\right)=0$.

Theorem 3.9: Let ${ }_{q} X$ is a $q$-normed space. Then $X$ is complete if and only if every a $q$-nest of non-empty $q$-closed sets has a non-empty intersection.

Proof: Suppose that ${ }_{q} X$ is complete and $\left\{E_{n}\right\}, n \in N$ be any nest of closed sets. If $x_{n} \in E_{n}, n \in N$ then we have that $\left\{x_{n}\right\}$ is a $q$-Cauchy sequence in ${ }_{q} X$, since

$$
{ }_{q}\left\|x_{n+k}-x_{n}\right\| \leq{ }_{q} d\left(E_{n}\right)
$$

holds for each $n$ and $k$. Thus there exists $x \in{ }_{q} X$ such that

$$
\lim _{n \rightarrow \infty}\left\|x_{n}-x\right\|=0
$$

and, we claim that $x \in \bigcap_{n=1}^{\infty} E_{n}$. Indeed, since $x_{m} \in E_{n}$ for $m \geq n$, we get $x \in{ }_{q} \overline{E_{n}}$ for each $n$. But since each $E_{n}$ is closed, ${ }_{q} \overline{E_{n}}=E_{n}$ holds. Therefore, $x \in E_{n}$ for each $n$ and this implies that $x \in \bigcap_{n=1}^{\infty} E_{n}$. Hence, $\bigcap_{n=1}^{\infty} E_{n} \neq \phi$.

Conversely, let every $q$-nest of $q$-closed sets has a non-empty intersection, and let $\left\{x_{n}\right\}$ be any $q$-Cauchy sequence in ${ }_{q} X$. Take $n_{1}$ such that

$$
{ }_{q}\left\|x_{n}-x_{n_{1}}\right\|<\frac{1}{2}, n>n_{1}
$$

Let, $E_{1}=\left\{x \mid{ }_{q}\left\|x-x_{n_{1}}\right\| \leq 1\right\}$. Take $n_{2}>n_{1}$ such that

$$
{ }_{q}\left\|x_{n}-x_{n_{2}}\right\|<\frac{1}{4}, n>n_{2} \text {. }
$$

Let $E_{2}=\left\{x \mid{ }_{q}\left\|x-x_{n_{2}}\right\| \leq \frac{1}{2}\right\}$. Then, $E_{2} \subset E_{1}$.

If we continue in this process, we obtain $n_{1}<n_{2}<n_{3}<\ldots$ and $E_{1} \supset E_{2} \supset E_{3} \supset \ldots$ with $\lim _{n \rightarrow \infty} q d\left(E_{n}\right)=0$. Hence,

$\bigcap_{n=1}^{\infty} E_{n} \neq \phi$, actually $\bigcap_{n=1}^{\infty} E_{n}=\{x\}$. Also, we see that ${ }_{q}\left\|x_{n_{k}}-x\right\| \rightarrow 0$ as $k \rightarrow \infty$, so $\left\{x_{n}\right\}$ has a $q$-convergent subsequence. Then by lemma 2.14 (3), we have

$$
{ }_{q}\left\|x_{n}-x\right\| \rightarrow 0 \text { as } n \rightarrow \infty \text {. }
$$

i.e. $\left\{x_{n}\right\} q$-converges, so ${ }_{q} X$ is complete.

Definition 3.10: Let $\left\{G_{\alpha}\right\}$ be a collection of open subsets of a $q$-normed space ${ }_{q} X$ and let $E$ be a subset of ${ }_{q} X$ such that 


$$
E \subseteq \bigcup_{\alpha \in A} G_{\alpha}
$$

where $A$ is the index set, then $\left\{G_{\alpha}\right\}$ is called a $q$-open cover of $E$ in ${ }_{q} X$. Any sub-collection of $\left\{G_{\alpha}\right\}$ which covers $E$ is called a sub-cover of $E$.

Definition 3.11: Let $X$ be a $q$-normed space. $A$ set $E \subseteq{ }_{q} X$ is said to be totally $q$-bounded if for every $\varepsilon>0$ there exists a finite number of points $x_{1}, x_{2}, \ldots, x_{n}$ such that

$$
E \subseteq \bigcup_{i=1}^{n} B_{\varepsilon}\left(x_{i}\right)
$$

If $E$ is a totally $q$-bounded set, then $\left(E,{ }_{q}\|\cdot\|\right)$ or simply $E$ is referred to as a totally $q$-bounded in $q$-normed space.

Lemma 3.12: A totally $q$-bounded subset of a $q$-normed space is a $q$-bounded set, but not conversely, in general.

Proof: Suppose $E$ is a totally $q$-bounded set of a $q$-normed space ${ }_{q} X$. For each $x_{i}, i=1,2, \ldots, n$ such that $x_{i} \in E$, the set ${ }_{q} B=\left\{x_{1} \ldots, x_{n}\right\}$ is a finite subset of $E$. By definition 3.11, every $x \in{ }_{q} B$ has a $q$-open ball of radius $\varepsilon$, where $\varepsilon>0$. Thus, $E$ can be decomposed into a finite number of $q$-bounded sets of $E$. Hence, $E$ is $q$-bounded. For the converse, we consider the following example:

Example 3.13: Let $E$ be the subset of the space $\ell_{2}$ consisting of the following points:

$$
x_{1}=\{1,0,0, \ldots\}, x_{2}=\{0,1,0, \ldots\}, x_{3}=\{0,0,1, \ldots\}, \ldots
$$

We note that $\forall x_{i} \in E, i=1,2, \ldots, q\left\|x_{i}\right\|=1$. Hence, $E$ is $q$-bounded.

Observe that ${ }_{q}\left\|x_{i}-x_{j}\right\|=\sqrt{2}$ if $i \neq j$. Thus,

$$
d(E)=\sup \left\{{ }_{q}\left\|x_{i}-x_{j}\right\| / x_{i}, x_{j} \in E\right\}=\sqrt{2},
$$

On the other hand, $E$ is not totally $q$-bounded. Indeed,

if $\varepsilon=1 / 2$, then the only non-empty subsets of $E$ with $q$-diameter less than $\varepsilon$ are the singleton sets.

Accordingly, the infinite set $E$ can not be decomposed into a finite number of disjoint subsets each with diameter less than $1 / 2$. Therefore, $\mathrm{E}$ is not totally bounded. Now, we introduce the concept of $q$-compact sets.

Definition 3.14: Let ${ }_{q} X$ be a $q$-normed space. A set $E \subseteq{ }_{q} X$ is said to be $q$-compact if every $q$-open cover of $E$ has a finite subcover. If $E$ is a $q$-compact set, then $\left(E,{ }_{q}\|\|.\right)$ or simply $E$ is referred to as a $q$-compact in $q$-normed space.

Lemma 3.15: Let ${ }_{q} X$ be a $q$-normed space. Then a $q$-compact subset of ${ }_{q} X$ is totally $q$-bounded, but not conversely, in general.

Proof: Let $E$ be a $q$-compact subset of ${ }_{q} X$ Then for every $\varepsilon>0$, the collection $\left\{{ }_{q} B_{\varepsilon}(x) \mid x \in E\right\}$ is a $q$-open cover of $E$ which has a finite sub-collection of $q$-open balls covers $E$. Hence by definition $3.11, E$ is a totally $q$-bounded set. In order to show that the converse is not true, consider the following example:

Example 3.16: Let the subset $E=(0,1)$ of $R$ with the $q$-norm

$$
{ }_{q}\|x\|=|x|, \forall x \in E \text {. }
$$

Since the $q$-open set $E$ can be decomposed into a finite number of $q$-open balls, $E$ is totally $q$-bounded [1]. But, $E$ is not $q$ compact. Indeed, let $\left\{G_{n}\right\}, n \in N$ be the collection of $q$-open intervals such that 


$$
G_{n}=\left(\frac{1}{n+2}, \frac{1}{n}\right) \quad, \forall n \in N
$$

Observe that $E=\bigcup_{n=1}^{\infty} G_{n}$, hence $\left\{G_{n}\right\}$ is a $q$-open cover of $E$. But, $\left\{G_{n}\right\}, n \in N$ contains no finite $q$-sub-cover. Indeed, let

$$
\left\{G_{m}\right\}=\left\{\left(x_{1}, y_{1}\right), \ldots,\left(x_{m}, y_{m}\right)\right\},
$$

be any finite sub-collection of $\left\{G_{n}\right\}$. If

$$
\varepsilon=\min \left(x_{1}, \ldots, x_{m}\right),
$$

then $\varepsilon>0$ and

$$
\left(x_{1}, y_{1}\right) \bigcup \ldots \bigcup\left(x_{m}, y_{m}\right) \subset(\varepsilon, 1) .
$$

But, $(0, \varepsilon]$ and $(\varepsilon, 1)$ are disjoint, hence $\left\{G_{m}\right\}$ is not a $q$-open cover of $E$, and so $E$ is not $q$-compact.

Theorem 3.17: If ${ }_{q} X$ is a $q$-compact in $q$-normed space then it is complete, but not conversely, in general.

Proof: Let ${ }_{q} X$ be compact and $\left\{E_{n}\right\}, n \in N$ be a $q$-nest of $q$-closed sets. We now show that ${ }_{q} X$ is complete by contradiction. Suppose ${ }_{q} X$ is not complete. Then by theorem 3.9, $\bigcap_{n=1}^{\infty} E_{n}=\phi$. Since ${ }_{q} X$ is $q$-compact, the collection $\left\{E_{n}^{c}\right\}, n \in N$ is a $q$-open cover of ${ }_{q} X$ contains a $q$-sub-cover of ${ }_{q} X$, where $E_{n}^{c}, n \in N$ is a $q$-open set by lemma 3.3. Then, we see that one of the sets $E_{n}, n \in N$ would be empty, which it is not true to our assumption. Hence, ${ }_{q} X$ is complete. For the converse, we consider the following example:

Example 3.18: The real line $R$ with the $q$-norm ${ }_{q}\|x\|=|x|, \forall x \in R$ is a complete $q$-normed space by remark 2.21. But, $R$ is not a $q$-compact space [17].

Definition 3.19: Let $X$ be a $q$-normed space. A set $E \subseteq{ }_{q} X$ is said to be sequentially $q$-compact if every sequence in $E$ contains a subsequence which $q$-converges to a point in $E$. If $E$ is a sequentially $q$-compact set, then $\left(E,{ }_{q}\|\cdot\|\right)$ or simply $E$ is referred to as a sequentially $q$-compact in $q$-normed space.

Theorem 3.20: A $q$-normed space ${ }_{q} X$ is sequentially $q$-compact if and only if it is complete and totally $q$-bounded.

Proof: Suppose that is sequentially $q$-compact. Firstly, we prove that $X$ is totally $q$-bounded. Let $x_{1} \in{ }_{q} X$ and take $\varepsilon>$ 0 . If

$$
{ }_{q}\left\|x_{1}-x\right\|<\varepsilon
$$

for all $x \in{ }_{q} X$ then we have a finite set $\left\{x_{1}\right\}$ such that ${ }_{q} X=B_{\varepsilon}\left(x_{1}\right)$. Otherwise there exists $x_{2}$ such that

$$
{ }_{q}\left\|x_{1}-x_{2}\right\| \geq \varepsilon \text {. }
$$

Similarly, if

$$
{ }_{q}\left\|x_{1}-x\right\|<\varepsilon \text { or }{ }_{q}\left\|x_{2}-x\right\|<\varepsilon,
$$

for all $x \in{ }_{q} X$, then we have a finite set $\left\{x_{1}, x_{2}\right\}$ such that

$$
{ }_{q} X={ }_{q} B_{\varepsilon}\left(x_{1}\right) \bigcup_{q} B_{\varepsilon}\left(x_{2}\right) .
$$

Otherwise there exists $x_{3}$ such that 


$$
{ }_{q}\left\|x_{1}-x_{3}\right\| \geq \varepsilon \text { and }{ }_{q}\left\|x_{2}-x_{3}\right\| \geq \varepsilon .
$$

Continuing in this manner, we arrive at a sequence $\left\{x_{1}, x_{2}, \ldots\right\}$ with the property

$$
{ }_{q}\left\|x_{i}-x_{j}\right\| \geq \varepsilon \text { for } i \neq j .
$$

But, a sequence $\left\{x_{n}\right\}$ has no $q$-convergent subsequence, contrary to sequential $q$-compactness. Therefore, we must have a finite points $\left\{x_{1}, x_{2}, \ldots, x_{n}\right\}$ such that

$$
{ }_{q} X=\bigcup_{i=1}^{n} B_{\varepsilon}\left(x_{i}\right) \text {. }
$$

i.e. ${ }_{q} X$ is totally $q$-bounded. Secondly, we prove that ${ }_{q} X$ is complete. Let $\left\{x_{n}\right\}$ be a $q$-Cauchy sequence in ${ }_{q} X$. Since ${ }_{q} X$ is sequentially $q$-compact, $\left\{x_{n}\right\}$ contains a subsequence $\left\{x_{n_{k}}\right\}$ which $q$-converges to a point in ${ }_{q} X$, call it $x$. But, by lemma 2.14 (3) we have $\left\{x_{n}\right\} q$-converges to $x$. Hence, ${ }_{q} X$ is complete.

Conversely, let ${ }_{q} X$ is complete and totally $q$-bounded. We must prove ${ }_{q} X$ is sequentially $q$-compact. Let $\left\{x_{n}\right\}$ be a sequence in ${ }_{q} X$. By total $q$-boundedness there is a finite collection of $q$-open balls of radius 1 and at least one of these open balls contains a subsequence $\left\{x_{n}^{(1)}\right\}$ of $\left\{x_{n}\right\}$. Denote for this $q$-open ball by $B_{1}$, of radius 1 . Also, there is a finite collection of $q$-open balls of radius $1 / 2$ and so there exists $B_{2}$, of radius $1 / 2$ which contains a subsequence $\left\{x_{n}^{(2)}\right\}$ of $\left\{x_{n}^{(1)}\right\}$. If we continue in this process then we obtain that the subsequence $\left\{x_{n}^{(1)}, x_{n}^{(2)}, \ldots\right\}$. This sequence is a $q$-Cauchy sequence, for $m, n \geq N$ implies $x_{n}^{(n)}, x_{m}^{(m)} \in B_{N}$. Since ${ }_{q} X$ is complete, we have $\left\{x_{n}^{(n)}\right\} q$-convergent. Hence ${ }_{q} X$ is sequentially $q$-compact.

Theorem 3.21: A $q$-normed space is $q$-compact if and only if it is sequentially $q$-compact.

Proof: Suppose that ${ }_{q} X$ is a $q$-compact in $q$-normed space. By theorem 3.17 and lemma $3.15,{ }_{q} X$ is complete and totally $q$-bounded. Hence by theorem 3.20 we have that ${ }_{q} X$ is sequentially $q$-compact.

Conversely, suppose that ${ }_{q} X$ is a sequentially $q$-compact $q$-normed space. We now show that ${ }_{q} X$ is $q$-compact by contradiction. Suppose that ${ }_{q} X$ is not $q$-compact. Then there exists a cover $\left\{G_{\alpha}\right\}$ of ${ }_{q} X$ which has no finite subcover. Since ${ }_{q} X$ is totally $q$-bounded, we have for each $n$, a cover of ${ }_{q} X$ consisting a finite number of open balls of radius $1 / \mathrm{n}$.

Thus, for each $n=1,2, \ldots$ at least one of the $q$-open balls of radius $1 / n$, call it ${ }_{q} B_{1 / \mathrm{n}}$, can not be covered by finitely many of the sets of $\left\{G_{\alpha}\right\}$.

Let $x_{n}$ be the center of ${ }_{q} B_{1 / \mathrm{n}}(x)$. Then ${ }_{q} X$ which is sequentially $q$-compact implies that $x_{n}$ has a subsequence $\left\{x_{n_{k}}\right\}$ $q$-converges to a point in ${ }_{q} X$, call it $x$. Since $\left\{G_{\alpha}\right\}$ covers ${ }_{q} X$, we have $x \in G_{\alpha}$ for some $\alpha$ and so the $q$-openness of $G_{\alpha}$ implies the existence of ${ }_{q} B_{r}(x) \subset G_{\alpha}$.

Now, by taking $k$ large enough we can ensure that ${ }_{q} B_{1 / n_{k}}\left(x_{n_{k}}\right) \subset{ }_{q} B_{r}(x)$. Hence the $q$-open ball ${ }_{q} B_{1 / n_{k}}\left(x_{n_{k}}\right) \subset G_{\alpha}$ for some $\alpha$ which is contrary to the fact that ${ }_{q} B_{1 / n_{k}}$ can not be covered by finitely many of the sets of $\left\{G_{\alpha}\right\}$. Thus, ${ }_{q} X$ must be $q$-compact.

Theorem 3.22: Every $q$-compact subset of a $q$-normed space is $q$-bounded and $q$-closed, but the converse may not be true.

Proof: Suppose that $E$ is a $q$-compact subset of a $q$-normed space ${ }_{q} X$, we prove that $E$ is $q$-bounded. Since $E$ is $q$ compact, so is totally $q$-bounded by lemma 3.15. Therefore, by lemma 3.12 , we have that $E$ is $q$-bounded. Now, we went to prove that $E$ is a $q$-closed set. If $x \in \bar{E}$, then by theorem 3.4 (a) there exists a sequence $\left\{x_{n}\right\}$ such that 


$$
x_{n} \rightarrow x \text { as } n \rightarrow \infty \text {. }
$$

Since $E$ is $q$-compact, so is sequentially $q$-compact by theorem 3.21 Hence by definition 3.19 , $\left\{x_{n}\right\}$ contains a subsequence $q$-converges to a point in $E$, call it $x$. Thus, $x \in E$. Therefore, $E$ is a $q$-closed set. In order to show that the converse is not true, we take the example 3.13. The set $E$ in this example is $q$-bounded. Also, we can observe that $\bar{E}=E$, because it does not contain any limit points, hence $E$ is $q$-closed. For the same reason, $E$ is not sequentially $q$ compact which implies that $E$ is not $q$-compact.

Definition 3.23: A subset $E$ of a $q$-normed space ${ }_{q} X$ is called relatively $q$-compact if its closure $\bar{E}$ is $q$-compact.

Remark 3.24: A compact set $E$ of a $q$-normed space ${ }_{q} X$ is relatively $q$-compact, because it is $q$-closed. But the converse may not be true, as it is shown in the example 3.16. In this example, the set $E=(0,1)$ is not $q$-compact. But, it is relatively $q$-compact. Indeed, since $\bar{E}=[0,1]$ which is $q$-closed set, $\bar{E}$ is sequentially $q$-compact by theorem 3.4 (b) and definition 3.19. Hence, by theorem 3.21 we have that $\bar{E}$ is $q$-compact. Therefore, $E$ is relatively $q$-compact.

\section{Quasi-Compact Operators}

In this section, we begin with a definition of the $q$-operator concept which is of many types and prove some results concerning this concept.

Definition 4.1: Let ${ }_{q} X$ and ${ }_{q} Y$ be $q$-normed spaces.

(a) A mapping $T$ from ${ }_{q} X$ into ${ }_{q} Y$ is called an $q$-operator. The value of $T$ at $\quad x \in{ }_{q} X$ is denoted by $T(x)$ or $T x$.

(b) $T$ is called a linear $q$-operator if the following condition holds:

$$
T(\alpha x+\beta y)=\alpha T x+\beta T y,
$$

for all $x, y \in{ }_{q} X$, and all scalars $\alpha, \beta$.

(c) $T$ is called $q$-continuous at a point $x_{0} \in{ }_{q} X$ if given $\varepsilon>0$, there exists a $\delta>0$, depending on $\varepsilon$ and $x_{0}$, such that

$$
{ }_{q}\left\|T x-T x_{o}\right\|<\varepsilon
$$

whenever

$$
{ }_{q}\left\|x-x_{o}\right\|<\delta \quad \forall x \in X .
$$

$T$ is called $q$-continuous on ${ }_{q} X$ if it is $q$-continuous at every point of ${ }_{q} X$.

(d) The $q$-operator $T$ is called $q$-bounded if there exists a positive real number $M$ such that

$$
{ }_{q}\|T x\| \leq M_{q}\|x\|, \quad \forall x \in{ }_{q} X .
$$

(e) ${ }_{q}\|T\|=\sup _{\substack{x \in X \\ x \neq 0}} \frac{\|T x\|}{q\|x\|}$ is called the $q$-norm of the $q$-bounded $q$-operator.

(f) For the $q$-operator $T$, the set $R(T)=\left\{T(x) \in Y \mid x \in{ }_{q} X\right\}$ and the set

$$
N(T)=\{x \in X \mid T(x)=0\}
$$

are called the range and null spaces of $T$ respectively.

Examples 4.2: Let ${ }_{q} X$ and ${ }_{q} Y$ be quasi-normed spaces.

(1) The zero $q$-operator $0:{ }_{q} X \longrightarrow{ }_{q} Y$ is defined by $O x=0$ for all $x \in{ }_{q} X$. This $q$-operator is a $q$-bounded linear operator and has the $q$-norm ${ }_{q}\|O\|=0$. 
(2) The identity $q$-operator $\quad I:{ }_{q} X \longrightarrow{ }_{q} X$ is defined by $I x=x \quad$ for all $x \in{ }_{q} X$. $I$ is a $q$-bounded linear operator and ${ }_{q}\|I\|=1$.

A necessary and sufficient condition for a linear $q$-operator to be $q$-continuous will be given in the following theorem:

Theorem 4.3: A linear $q$-operator from a $q$-normed space into a $q$-normed space is $q$-continuous if and only if it is $q$ bounded.

Proof: Let $T:{ }_{q} X \longrightarrow{ }_{q} Y$ is a $q$-linear operator, where ${ }_{q} X$ and ${ }_{q} X$ are $q$-normed spaces. For $T=0$, the theorem is trival. If $T \neq 0$, then ${ }_{q}\|T\| \neq 0$. Suppose $T$ is $q$-bounded and consider any $x_{0} \in{ }_{q} X$. Let any $\varepsilon>0$ be given. Then, since $T$ is $q$-linear, for every $x \in{ }_{q} X$ such that

$$
{ }_{q}\left\|x-x_{0}\right\|<\delta \text { where } \delta=\frac{\varepsilon}{\|T\|}
$$

we obtain:

$$
{ }_{q}\left\|T x-T x_{0}\right\|={ }_{q}\left\|T\left(x-x_{0}\right)\right\| \leq_{q}\|T\|_{q}\left\|x-x_{0}\right\|<{ }_{q}\|T\| \delta=\varepsilon .
$$

Since $x_{0} \in{ }_{q} X$ was arbitrary, $T$ is $q$-continuous.

Conversely, let $T$ is $q$-continuous at an arbitrary $x_{0} \in{ }_{q} X$. Then, given any $\varepsilon>0$, there is a $\delta>0$ such that:

$$
{ }_{q}\left\|T x-T x_{0}\right\|=\leq \varepsilon, \text { whenever, } \forall x \in_{q} X
$$

Now, take any $y \neq 0$ and put

$$
x=x_{o}+\frac{\delta}{\|y\|} y \text {, or } x-x_{o}=\frac{\delta}{\|y\|} y .
$$

Hence, ${ }_{q}\left\|x-x_{0}\right\|=\delta$. Using inequality (35) and since $T$ is linear, we have:

$$
\begin{gathered}
{ }_{q}\left\|T x-T x_{o}\right\|={ }_{q}\left\|T\left(x-x_{o}\right)\right\| \\
={ }_{q}\left\|T\left(\frac{\delta}{{ }_{q}\|y\|} y\right)\right\| \\
=\frac{\delta}{{ }_{q}\|y\|}{ }_{q}\|T y\| \leq \varepsilon
\end{gathered}
$$

Thus,

$$
{ }_{q}\|T y\| \leq \frac{\varepsilon}{\delta}{ }_{q}\|y\| .
$$

This can be written

$$
{ }_{q}\|T y\| \leq M_{q}\|y\|,
$$

where $M=\frac{\varepsilon}{\delta}$, and this shows that $T$ is $q$-bounded.

Definition 4. 4: Let ${ }_{q} X$ and ${ }_{q} Y$ be two $q$-normed spaces.

(a) A q-operator $T:{ }_{q} X \longrightarrow{ }_{q} Y$ is said to be one-to-one if the following condition is satisfied: 
$T x_{1}=T x_{2}$ implies $x_{1}=x_{2}$, for every $x_{1}, x_{2} \in{ }_{q} X$.

(b) A q-operator $T$ is said to be onto if $R(T)={ }_{q} Y$

(c) A $q$-operator $T$ is said to be invertible if it is one-to-one and onto. That is, the inverse of $T$ exists which denotes by $T^{-1}$. Now, we need the following lemma whose proof can be found in [17]:

Lemma 4.5: The inverse of a linear $q$-operator $T$ exists if and only if $T x=0$ implies $x=0$. When $T^{-1}$ exists, it is a linear $q$-operator.

Lemma 3.6: Suppose $T$ is a linear $q$-operator from ${ }_{q} X$ into ${ }_{q} Y$, where ${ }_{q} X$ and ${ }_{q} Y$ are $q$-normed spaces. Then the inverse $T^{-1}$ exists and is $q$-continuous on ${ }_{q} X$ if and only if there exists a constant $M>0$ such that:

$$
M_{q}\|x\| \leq{ }_{q}\|T x\|
$$

for every $x \in{ }_{q} X$.

Proof: If inequality (40) holds and $T x=0$, it follows that $x=0$. So by lemma $4.5, T^{-1}$ exists. Now, $y=T x$ is equivalent to $x=T^{-1} y$. Hence inequality (40) is equivalent to

$$
M_{q}\left\|T^{-1} y\right\| \leq{ }_{q}\|y\|, \text { or }{ }_{q}\left\|T^{-1} y\right\| \leq \frac{1}{M}{ }_{q}\|y\|,
$$

for all $y$ in the range of $T$ which is the domain of $T^{-1}$. This implies that $T^{-1}$ is $q$-continuous by theorem 4.3.

Conversely, suppose that $T^{-1}$ exists and is $q$-bounded. Then,

$$
\forall x \in \in_{q} X, x=T^{-1} \mathrm{y}
$$

for all $y$ in the range of $T$ is equivalent to $y=T x$. Since $T^{-1}$ is $q$-bounded by theorem 4.3, we have

$$
{ }_{q}\left\|T^{-1} y\right\| \leq M_{1}{ }_{q}\|y\|
$$

where $M_{1}>0$. This implies that:

$$
\frac{1}{M_{1}}{ }_{q}\left\|T^{-1} y\right\| \leq{ }_{q}\|T x\| \Rightarrow \frac{1}{M_{1}}{ }_{q}\|x\| \leq{ }_{q}\|T x\| .
$$

If we set $=\frac{1}{M_{1}}$, then, we obtain inequality (40).

Remark 4.7: Let ${ }_{q} X$ and $Y$ be a $q$-normed spaces. Then ${ }_{q} B\left({ }_{q} X,{ }_{q} Y\right)$ denotes the set of all $q$-bounded linear operators from ${ }_{q} X$ into ${ }_{q} Y$. With the $q$-norm $\quad{ }_{q}\|T\|=\sup _{\substack{x \in q \\ x \neq 0}} \frac{\|T x\|}{{ }_{q}\|x\|}$ for all

$$
T \in{ }_{q} B\left({ }_{q} X,{ }_{q} Y\right)
$$

then,

$$
{ }_{q} B\left({ }_{q} X,{ }_{q} Y\right)
$$

is a $q$-normed space.

Remark 4.8: Let $T \in{ }_{q} B\left({ }_{q} X,{ }_{q} Y\right)$, where ${ }_{q} X$ is a $q$-normed space. If we multiply $T$ by itself $\mathrm{n}$-times. Then, 


$$
{ }_{q}\left\|T^{n}\right\| \leq{ }_{q}\|T\|^{n}, \forall n \in N
$$

Indeed, for any $x \in{ }_{q} X, \quad{ }_{q}\left\|T^{2} x\right\|={ }_{q}\|T(T x)\|$

$$
\begin{aligned}
& \leq_{q}\|T\|{ }_{q}\|T x\| \\
& \quad \leq{ }_{q}\|T\|^{2}\|x\|, \text { as } T \text { is } q \text {-bounded } \\
& \quad{ }_{q}\left\|T^{2}\right\|{ }_{q}\|T\|^{2}
\end{aligned}
$$

If we continue in this process, we obtain inequality (46).

Theorem 4.9: If $Y$ is a $q$-Banach space, then ${ }_{q} B\left({ }_{q} X,{ }_{q} Y\right)$ is a $q$-Banach space.

Proof: We consider an arbitrary $q$-Cauchy sequence $\left\{T_{n}\right\}$ in ${ }_{q} B\left({ }_{q} X,{ }_{q} Y\right)$ Then, for every $\varepsilon>0$ there is a positive integer $N$ such that

$$
{ }_{q}\left\|T_{n}-T_{m}\right\|<\varepsilon, \text { where } m, n \geq N \text {. }
$$

By remark 4.7, and for $x \in{ }_{q} X$ and $m, n \geq N$, we obtain:

$$
{ }_{q}\left\|T_{n} x-T_{m} x\right\|={ }_{q}\left\|\left(T_{n}-T_{m}\right) x\right\| \leq_{q}\left\|T_{n}-T_{m}\right\|{ }_{q}\|x\|<\varepsilon_{q}\|x\|
$$

Now, for any fixed $x$ and given $\hat{\varepsilon}$ we may choose $\varepsilon=\varepsilon_{x}$ so that

$$
\varepsilon_{x} \quad{ }_{q}\|x\|<\hat{\varepsilon} .
$$

Then, from inequality (47), we have

$$
{ }_{q}\left\|T_{n} x-T_{m} x\right\|<\hat{\varepsilon}
$$

and see that $\left\{T_{n} x\right\}$ is a $q$-Cauchy sequence in ${ }_{q} Y$. Since ${ }_{q} Y$ is complete, $\left\{T_{n} x\right\}$-converges, say $\left\{T_{n} x\right\} \rightarrow y$.

Clearly, the limit $y \in{ }_{q} Y$ depends on the choice of $x \in{ }_{q} X$. This defines an $q$-operator $T:{ }_{q} X \longrightarrow{ }_{q} Y$, where $y=$ $T x$. The $q$-operator $T$ is linear, because

$$
\begin{gathered}
\operatorname{Lim}_{n \rightarrow \infty} T_{n}(\alpha x+\beta z)=\operatorname{Lim}_{n \rightarrow \infty}\left(\alpha T_{n} x+\beta T_{n} z\right) \\
=\alpha \operatorname{Lim}_{n \rightarrow \infty} T_{n} x+\beta \operatorname{Lim}_{n \rightarrow \infty} T_{n} z
\end{gathered}
$$

for all $x, z \in \in_{q} X$ and for any scalers, $\alpha$ and $\beta$. Now, we prove that $T$ is $q$-bounded and $T_{n} \rightarrow T$. That is ${ }_{q}\left\|T_{n}-T\right\| \rightarrow 0$.

Since inequality (47) holds for every $m \geq N$ and $T_{m} x \rightarrow T x$, we may let $m \rightarrow \infty$. Using the $q$-continuity of the $q$-norm, we then obtain from (47) for every $n \geq N$ and $x \in{ }_{q} X$,

$$
{ }_{q}\left\|T_{n} x-T x\right\|={ }_{q}\left\|T_{n} x-\lim _{m \rightarrow \infty} T_{m} x\right\|=\lim _{m \rightarrow \infty}\left\|T_{n} x-T_{m} x\right\| \leq \varepsilon \quad{ }_{q}\|x\| .
$$

This shows that $\left(T_{n}-T\right)$ with $n \geq N$ is a $q$-bounded linear operator. Since $T_{n}$ is $q$-bounded, $T=T_{n}-\left(T_{n}-T\right)$ is $q$ bounded. That is, $T \in{ }_{q} B\left({ }_{q} X,{ }_{q} Y\right)$. Furthermore, if we take the supremum over all $x$ of $q$-norm in inequality (50) is 1 , we obtain

$$
{ }_{q}\left\|T_{n}-T\right\| \leq \varepsilon, n \geq N .
$$

Hence, 


$$
{ }_{q}\left\|T_{n}-T\right\| \rightarrow 0
$$

Since $\left\{T_{n}\right\}$ was arbitrary $q$-Cauchy sequence, ${ }_{q} B\left({ }_{q} X,{ }_{q} Y\right)$ is a $q$-Banach space.

Lemma 4.10: Let $T \in{ }_{q} B\left({ }_{q} X,{ }_{q} X\right)$ where ${ }_{q} X$ is a $q$-Banach space. If ${ }_{q}\|T\|<1$, then $(I-T)^{-1}$ exists as a $q$ bounded linear $q$-operator on ${ }_{q} X$ and,

$$
(I-T)^{-1}=\sum_{i=0}^{\infty} T^{i}=I+T+T^{2}+\ldots
$$

where the series on the right is $q$-convergent in the space ${ }_{q} B\left({ }_{q} X,{ }_{q} X\right)$ Furthermore,

$$
{ }_{q}\left\|(I-T)^{-1}\right\| \leq c\left(\frac{1}{1-{ }_{q}\|T\|}\right), \text { where } c \geq 1 \text {. }
$$

Proof: Since ${ }_{q}\|T\|<1$, the geometric series $\sum_{i=0}^{\infty}{ }_{q}\|T\|^{i} q$-converges by lemma 2.23 But, by remark 4.8, we have that

$$
{ }_{q}\left\|T^{i}\right\| \leq{ }_{q}\|T\|^{i}, \forall i
$$

Hence by definition $2.23(\mathrm{~b})$, the series in (53) is absolutely $q$-convergent for ${ }_{q}\|T\|<1$. Since ${ }_{q} X$ is complete, so is ${ }_{q} B\left({ }_{q} X,{ }_{q} X\right)$ by theorem 4.9. Thus by theorem 2.19, absolute $q$-convergence implies $q$-convergence. If we let

$$
S=\sum_{i=0}^{\infty} T^{i}, \text { we see that } T S=S T=\sum_{i=0}^{\infty} T^{i+1},
$$

and hence

$$
(I-T) S=S(I-T)=I(55)
$$

This implies that $(I-T)^{-1}$ exists and is equal to $\mathrm{S}$. Now,

$$
\begin{aligned}
\left\|(I-T)^{-1}\right\| & ={ }_{q}\left\|\sum_{i=0}^{\infty} T^{i}\right\| \\
& \leq c\left(\sum_{i=0}^{\infty}{ }_{q}\|T\|^{i}\right), \text { where } c \geq 1 . \\
& =c\left(\frac{1}{1-{ }_{q}\|T\|}\right),
\end{aligned}
$$

because the series $\sum_{i=0}^{\infty}{ }_{q}\|T\|^{i} q$-converges. Thus, inequality (54) is satisfied. We now take the concept of $q$-compact operator.

Definition 4.11: A $q$-linear operator $T:{ }_{q} X \longrightarrow{ }_{q} Y$, where ${ }_{q} X$ and ${ }_{q} Y$ are $q$-normed spaces is said to be a $q$ compact $q$-operator if for every $q$ - bounded set $E$ of ${ }_{q} X$ its image $T(E)$ is relatively $q$-compact.

Theorem 4.12: Let ${ }_{q} X$ and ${ }_{q} Y$ be $q$-normed spaces and $T:{ }_{q} X \longrightarrow{ }_{q} Y$ a $q$-linear operator. Then $T$ is $q$-compact if and only if for every $q$-bounded sequence $\left\{x_{n}\right\}$ in ${ }_{q} X,\left\{T x_{n}\right\}$ has a $q$-convergent subsequence. 
Proof: Suppose that $T$ is $q$-compact and $\left\{x_{n}\right\}$ is a bounded sequence, then the closure of $\left\{T x_{n}\right\}$ in ${ }_{q} Y$ is $q$-compact. Hence by definition 3.19 , we have that $\left\{T x_{n}\right\}$ contains a $q$-convergent subsequence.

Conversely, suppose that every $q$-bounded sequence $\left\{x_{n}\right\}$ contains a subsequence $\left\{x_{n_{k}}\right\}$ such that $\left\{T x_{n_{k}}\right\} q$-converges in ${ }_{q} X$. Consider any $q$-bounded subset $E$ of ${ }_{q} X$, and let $\left\{y_{n}\right\}$ be any sequence in $T(E)$. Then $y_{n}=T x_{n}$ for some $x_{n}$ $\in E$, and $\left\{x_{n}\right\}$ is $q$-bounded, since $E$ is $q$-bounded. By assumption, $\left\{T x_{n}\right\}$ contains a $q$-convergent subsequence. Hence by definition 3,19 and theorem $3.21, \overline{T(E)}$ is $q$-compact because $\left\{y_{n}\right\}$ in $T(E)$ was arbitrary. Therefore by definition 3.11 , we have that $T$ is a $q$-compact operator.

\section{Conclusion}

In this paper, we have explored the notion $q$-compactness in a $q$-Banach space for $\ell_{p}, 0<p<1$. Then, we prove that the sequence space $\ell_{p}, 0<p<1$, is not compact normed space, but may be a $q$-compact in $q$-normed space. We present and examine many results concerning this notion. We have studied the relation between a $q$-compactness and a $q$-completeness in this space. Thus, we have shown that, there exists a space which is not compact Banach space, but may be a $q$-compact Banach space. Finally, we have been proved that the existence of an operator $T:{ }_{q} X \longrightarrow{ }_{q} Y$ is compact.

\section{References}

[1] Aliprantis C. and Burkinshaw O., "Principles of Real Analysis", Elsevier North Holland, Inc. 1998.

[2] Al-Jawari N., Al-Janabi A. and Al-Ehamedi, The quasi-Banach space $L_{p}$ for $0<p<1$ and its applications on infinitedimensional Leslie matrix, Al-Mustansriyah Journal of science, Vol. 19, No. 8, 2008.

[3] Al-Saphory R., Al-Janabi A. and Al-Delfi J., Quasi-Banach space for the sequence space $\ell_{p}$, where $0<p<1$, Journal of Education College, University of Al-Mustansriyah, Baghdad, Iraq, Vol. 1, No. 1, pp. 1-12, 2007.

[4] Al-Saphory R., Al-Janabi A. and Al-Delfi J., The quasi-Banach space $\ell_{p}$, for $0<p<1$ and its applications on infinite-dimensional Leslie matrix, Tikrit journal of pure science, Tikrit University, Iraq, submitted, 2012.

[5] Arnold D. and Yokoyama K., "The Leslie Matrix"., Math 45-linear Algebra, David-Arnold @ Eurka. redwoods.cc.ca.us, pp. 1-11.

[6] Bastero J., Bernués J. and Pina A., "An Extension of Milman's Reverse Brunn-Minkowski Inequality", Arxiv: math., FA / 9501210 V1, 1995.

[7] Curtain R. F. and Pritchard A. J., "Functional Analysis in Modern Applied Mathematics", Academic Press, Inc., London, 1977.

[8] Gupta K. P., "Topology", Pargati Prakashan Publishing Company, Delhi, India, 2000.

[9] Kalton N., Convexity, type and the three space problem, Studia Math., Vol. 69, pp. 247-287, 1981.

[10] Litavak A., On the constant in the reverse Brunn-Minkowski inequality for $p$-convex Balls, Convex Geometric Analysis, MSRI Publications, V0I. 34, 1998.

[11] Maddox I. J., "Elements of Functional Analysis", Cambridge University Press, 1988.

[12] Milman V., Inégalité de Brunn-Minkowski inverse et applications à la théorie locale des espaces normés, $C$. $R$. Acad. Sci. Paris Sér. Vol. 1, no. 302, pp. 25-28, 1986.

[13] Mukherij O., On an infinite-dimensional Leslie matrix in sequence space $\ell_{p}, 1 \leq p \leq \infty, P h$. D thesis, College of science, Al-Mustansriyah University, Iraq, 1998.

[14] Rudin W., "Principlies of Mathematical Analysis", MacGraw-Hill, Inc., Tokyo, Japan, 1976.

[15] Siddiqi A. H., "Functional Analysis With Applications", Tata MacGraw-Hill Publishing Company, Ltd, New Delhi, India, 1986.

[16] Sykes Z. M., "On Discrete Stable Population Theory", Biometrics, 25, PP. 285-293, 1969.

[17] Tayler A. "Introduction to Functional Analysis", John Wiley and Sons, Inc., New York, 1967.

[18] Kalton N., Quasi-Banach spaces, In: W.B. Johnson, J. Lindenstrauss (eds.), Handbook of the Geometry of Banach spaces, Vol. 2, North Holland, 1101-1129, 2003. 